\begin{tabular}{|c|l|}
\hline Title & The c-di-GMP recognition mechanism of the PilZ domain of bacterial cell lulose synthase subunit A \\
\hline Author(s) & Fujiwara, Takaaki; Komoda, Keisuke; Sakurai, Naofumi; Tajima, Kenji; Tanaka, Isao; Y ao, Min \\
\hline Citation & $\begin{array}{l}\text { Biochemical and Biophysical Research Communications, 431(4), 802-807 } \\
\text { https://doi.org/L0.1016/.bbrc.2012.12.103 }\end{array}$ \\
\hline Issue Date & 2013_02-22 \\
\hline Doc URL & http://hdl.handle.net/2115/52657 \\
\hline Type & article (author version) \\
\hline File Information & PilZ_BBRC_final.pdf \\
\hline
\end{tabular}

Instructions for use 


\section{The c-di-GMP recognition mechanism of the PilZ domain of bacterial cellulose synthase subunit A}

Takaaki Fujiwara ${ }^{1}$, Keisuke Komoda ${ }^{1,2}$, Naofumi Sakurai ${ }^{2}$, Kenji Tajima ${ }^{3}$, Isao $\operatorname{Tanaka}^{1,2}$, Min Yao ${ }^{1,2}$

${ }^{1}$ Graduate School of Life Science, Hokkaido University, Kita-10, Nishi-8, Kita-ku, Sapporo, 060-0810, Japan

${ }^{2}$ Faculty of Advanced Life Science, Hokkaido University, Kita-10, Nishi-8, Kita-ku, Sapporo, 060-0810, Japan

${ }^{3}$ Faculty of Engineering, Hokkaido University, Kita-13, Nishi-8, Kita-ku, Sapporo, 060-8628, Japan

* Corresponding author e-mail address: yao@castor.sci.hokudai.ac.jp Fax +81(11)706-4481; Tel +81(11)706-4481 


\section{Abstract}

In some Proteobacteria and Firmicutes such as Pseudomonas aeruginosa, Vibrio cholerae, Xanthomonas campestris, and Clostridium difficile, cyclic dimeric guanosine monophosphate (c-di-GMP) is known to regulate cellular processes, including motility, biofilm formation, and virulence, as a second messenger. Cellulose production in Acetobacter xylinum, a model organism of cellulose biosynthesis, also depends on cellular c-di-GMP level. In cellulose-synthesizing bacteria, a terminal complex localized in the cell membrane synthesizes cellulose and regulates the production of cellulose sensed by c-di-GMP. Although previous studies indicated that the PilZ domain conserved in cellulose synthase subunit A (CeSA) was part of a receptor for c-di-GMP, the recognition mechanism by PilZ domain of CeSA remains unclear. In the present study, we studied the interaction between c-di-GMP and the PilZ domain of CeSA from a structural viewpoint. First, we solved the crystal structure of the PilZ domain of CeSA from A. xylinum (AxCeSA-PilZ) at $2.1 \AA$ resolution. Then, comparison of the sequence and structure of AxCeSA-PilZ to those of known structures of PilZ, such as VCA0042, 
PP4397, and PA4608, indicated the involvement of Lys573 and Arg643 of AxCeSA-PilZ in the recognition of c-di-GMP besides the RxxxR motif. Finally, the binding characteristics of c-di-GMP to AxCeSA-PilZ and mutants were determined with isothermal titration calorimetry, indicating that the residues corresponding to Lys573 and Arg643 in AxCeSA-PilZ generally contribute to the binding of c-di-GMP to PilZ.

\author{
Abbreviations \\ c-di-GMP, cyclic dimeric guanosine monophosphate; ITC, isothermal titration \\ calorimetry; CeS, cellulose synthase; DGC, diguanylate cyclase; PDE, \\ phosphodiesterase; STING, stimulator of interferon genes
}

\title{
Keywords
}

X-ray crystallography; PilZ domain; c-di-GMP; bacterial cellulose synthase; binding stoichiometry; isothermal titration calorimetry; 


\section{Introduction}

Cyclic dimeric guanosine monophosphate (c-di-GMP) is a bacterial second messenger that controls a wide variety of cellular processes, such as biofilm formation [1], virulence [2], cell cycle [3], and motility [3,4]. In the c-di-GMP-dependent signaling cascades, c-di-GMP binds to different effectors, including transcription factors, degenerate GGDEF/EAL domain-containing proteins, PilZ domain-containing proteins, and even nucleotides; riboswitches [5,6]. The transcription factor FleQ from Pseudomonas aeruginosa controls expression of flagellar genes and the biofilm-promoting pel operon [1]. Another transcription factor, Clp, from the plant pathogen Xanthomonas campestris suppresses virulence gene expression by increased intracellular level of c-di-GMP coupled with a quorum sensing system [2]. In Caulobacter crescentus, PopA that has a degenerate GGDEF motif binds c-di-GMP and is localized around the cell pole to interact with the downstream components for controlling the cell cycle [3]. DgrA from C. crescentus, which is classified as a PilZ domain-containing protein, blocks cell motility linked to flagellar motor function upon c-di-GMP binding [4]. Interestingly, c-di-GMP receptor was found not only in bacteria 
but also in mammalian cells. Human STING (stimulator of interferon genes) was identified as a direct sensor of c-di-GMP, and recognition of c-di-GMP by STING induces an innate immune response through the type I interferon signaling pathway [7]. The cellular level of c-di-GMP itself is controlled by opposing activities of diguanylate cyclase (DGC) and phosphodiesterase (PDE). Two molecules of GTP are ligated to form c-di-GMP by the activity of the GGDEF domain of DGC [8], while it is linearized to dinucleotide 5'-pGpG by the EAL domain of PDE [9,10]. In addition, HD-GYP domain-containing enzymes, the other c-di-GMP specific PDE, degrade c-di-GMP into GMP via 5'-pGpG. The GGDEF domain of DGC and the EAL domain of PDE are generally distinguished from degenerate GGDEF/EAL domain-containing proteins serving as c-di-GMP effectors.

Among the various groups of effectors, PilZ domain-containing proteins or single PilZ proteins have been studied in detail, and the first verified receptor for c-di-GMP was determined by computational means [11]. Moreover, the structures of several PilZ proteins alone and in complex with c-di-GMP have been determined by NMR and X-ray crystallography [12 - 14]. VCA0042/PlzD from Vibrio cholerae (PDB: 1YLN) 
and PP4397 from Pseudomonas putida (PDB: 2GJG) have a C-terminal PilZ domain in conjunction with N-terminal YcgR-N domain. Interestingly, the ligand-binding stoichiometry and change in oligomeric state induced upon c-di-GMP binding are distinct from each other, although the same domain organization is employed in both proteins and conserved RxxxR and D/NxSxxG motifs are responsible for c-di-GMP binding. The structure of VCA0042 in complex with c-di-GMP showed that monomeric c-di-GMP was accommodated around the interface between the YcgR-N and PilZ domains [12], whereas binding of two molecules of c-di-GMP induces the dimer-to-monomer transition of PP4397 [13]. Structural analysis of PA4608 from $P$. aeruginosa, which is one of the single PilZ proteins (PDB: 2L74) [14], revealed that self-intercalated c-di-GMPs observed in the structure of holo-PP4397 were also seen in PA4608. Binding affinity and stoichiometry of c-di-GMP to VCA0042 or PP4397 were measured by isothermal titration calorimetry (ITC). The results of the experiments indicated that the residue just before the $\operatorname{RxxxR}$ motif affected the binding characteristics.

The membrane-associated cellulose synthase complex called terminal complex from 
Acetobacter xylinum contains at least three polypeptides, i.e., cellulose synthase subunit

(CeS) AB (or A and B in strain ATCC53582), C and D encoded within a single operon

[15]. Among them, the structure of CeSD has been determined and a role of CeSD in exporting synthesized glucan chain was proposed [16]. CeSA was shown to be a membrane protein with a glycosyltransferase domain and PilZ domain. Previous studies showed that the biosynthesis of cellulose in A. xylinum [17] and other cellulose-synthesizing bacteria [18] was actually promoted by the increased level of c-di-GMP. Moreover, c-di-GMP weakly $(30 \mu \mathrm{M})$ bound to the PilZ domain conserved in the C-terminal region of bacterial CeSA in vitro [19]. However, the mechanism of recognition by the PilZ domain of CeSA is poorly understood.

In this study, we determined the crystal structure of PilZ domain of CeSA from $A$. xylinum (AxCeSA-PilZ). Furthermore, binding experiments using ITC were carried out to characterize the binding of c-di-GMP to AxCeSA-PilZ and mutants. Based on the results of the binding experiments, we propose a binding mechanism shared by the PilZ domain-containing proteins. 


\section{Materials and methods}

2.1 Cloning, expression, and purification

AxCeSA-PilZ gene sequence fragment was amplified by PCR from A. xylinum ATCC23769 strain genome using the specific primers, 5'-GAATTCCATATGCGGGATCCGCAGAAACGCAACAGTC-3' and 5'-CCGCTC-

GAGGCCGAACACCAG-3'. The amplified fragment DNAs digested with BamHI and XhoI were ligated into pET-26b (Novagen). The resulting construct included cloning artifacts derived from restriction enzyme sites and a C-terminal hexahistidine tag. The expression plasmid consisting of AxCeSA-PilZ coding sequence was introduced in Escherichia coli strain BL21 (DE3) by electroporation. Cells were grown overnight at $37^{\circ} \mathrm{C}$ in $5 \mathrm{~mL}$ of pre-culture medium consisting of Luria broth (LB) with $25 \mu \mathrm{g} \mathrm{mL}{ }^{-1}$ kanamycin and the cell culture was inoculated into $1 \mathrm{~L}$ of $\mathrm{LB}$ medium with the same concentration of kanamycin. When the cell density reached an $\mathrm{OD}_{600}$ of 0.5 , isopropyl $\beta$-D-1-thiogalactopyranoside (IPTG) was added to the medium to a final concentration of $0.5 \mathrm{mM}$ for induction. The cells were cultured overnight at $25^{\circ} \mathrm{C}$ and were then harvested by centrifugation at $4500 \times g$ for $15 \mathrm{~min}$ at $4^{\circ} \mathrm{C}$ and rinsed with $50 \mathrm{mM}$ 
Tris- $\mathrm{HCl}$ buffer $(\mathrm{pH} 8.0)$ and $300 \mathrm{mM} \mathrm{NaCl}$ before being stored at $-80^{\circ} \mathrm{C}$ until use. The frozen cells resuspended in $50 \mathrm{mM}$ Tris- $\mathrm{HCl}$ buffer $(\mathrm{pH} 8.0)$ and $300 \mathrm{mM} \mathrm{NaCl}$ were disrupted by sonication. After the lysate was centrifuged at $40000 \times g$ for $15 \mathrm{~min}$ at $10^{\circ} \mathrm{C}$ to remove cell debris, the supernatant was applied to a HisTrap HP $1 \mathrm{~mL}$ column (GE Healthcare). The eluate containing the proteins of interest was collected and further purified on a Superdex-75 16/60 column (GE Healthcare) equilibrated by a solution containing $50 \mathrm{mM}$ MES-NaOH buffer (pH 6.0) and $150 \mathrm{mM} \mathrm{NaCl}$.

\subsection{Crystallization}

The purified AxCeSA-PilZ was concentrated by ultrafiltration using Vivaspin 6 with a 5000 molecular weight cutoff membrane (GE Healthcare) to a final concentration of 5 $\mathrm{mg} \mathrm{mL}^{-1}$. The initial screening of the crystallization conditions of AxCeSA-PilZ was performed using The JCSG Core Suites I, II, III, and IV (Qiagen) by the sitting-drop vapor-diffusion method. A drop was made by mixing $0.5 \mu \mathrm{L}$ of the solution containing proteins of interest and an equal volume of a reservoir solution and equilibrated at $20^{\circ} \mathrm{C}$. Optimization of crystallization conditions was then performed by the hanging-drop 
vapor-diffusion method at $20^{\circ} \mathrm{C}$. The crystals were grown in the drop mixed with 1.0 $\mu \mathrm{L}$ of a protein solution and $1.0 \mu \mathrm{L}$ of a reservoir solution. The single crystals of AxCeSA-PilZ suitable for X-ray diffraction experiments were obtained from the drop containing 0.1 M phosphate-citrate buffer (pH 3.8) and 30\% (v/v) PEG300.

2.3 X-ray diffraction data collection, structural determination, and refinement

A crystal of AxCeSA-PilZ was picked up and transferred into crystallization solution supplemented with $15 \%(\mathrm{v} / \mathrm{v})$ glycerol as a cryoprotectant. Crystals soaked into the solution were flash-cooled under a nitrogen gas stream. X-ray diffraction images of the crystal were collected using an ADSC Quantum 210r CCD detector with synchrotron radiation at a wavelength of $1.0 \AA$ on the BL-5A beamline of Photon Factory (Tsukuba, Japan). Collected diffraction data set of AxCeSA-PilZ was processed using the XDS program suite [20]. Data collection statistics are summarized in Table 1. The structure of AxCeSA-PilZ was then determined by the molecular replacement method using AutoMR in Phenix [21] with the structure of Se-Met substituted for AxCeSA-PilZ as a search model. Rotation and translation functions were calculated using data of $45-3.0$ 
$\AA$ resolution. Refinement of the structure of $A x C e S A-P i l Z$ was carried out by iterative cycles of phenix.refine in Phenix and real-space refinement in COOT [22]. All refinement statistics evaluated by the program MolProbity [23] are listed in Table 1. The coordinates of AxCeSA-PilZ have been deposited in the RCSB Protein Data Bank with accession code 4I86.

2.4 Site-directed mutagenesis

Lys573 was replaced with valine (K573V) or alanine (K573A), while Arg643 was replaced with tryptophan (R643W) or alanine (R643A) based on the QuikChange technique. QuikChange PCR works using a pair of complementary primers and AxCeSA-PilZ gene fragment ligated into the pET-26b vector as template DNA. Primers, 5'-CGGGATCCGCAGGTACGCAAC-3' and 5'-CGATGACTGTTGCGTACCTGCG-

G-3' were used for K573V, 5'-CGGGATCCGCAGGCTCGCAAC-3' and 5'-CGATGACTGTTGCGAGCCTGCGG-3' were used for K573A, 5'-GCCAGGATCATCTGGGCTGGC-3' and 5'-GCCCGTTGCCAGCCCAGATG-3' were used for R643W, 5'-GCCAGGATCATCGCAGCTGGC-3' and 
5'-GCCCGTTGCCAGCTGCGATG-3' were used for R643A. The resulting DNA pool was then treated with $D p n \mathrm{I}$ at $37^{\circ} \mathrm{C}$ for $1 \mathrm{~h}$. Each plasmid was introduced in $E$. coli XL10-Gold by heat shock and extracted from the cultured cells. All mutations were confirmed by DNA sequencing. Cells of BL21 (DE3) were transformed with the mutant plasmid. Mutants were expressed and purified according to procedures similar to those used for wild-type $A x$ CeSA-PilZ for the binding experiment. The purity of the proteins was evaluated by SDS-PAGE followed by Coomassie Brilliant Blue R-250 staining.

\subsection{Binding experiments with ITC}

The proteins were quantified by Bradford assay (Bio-Rad). Measured concentration of wild-type AxCeSA-PilZ, mutants K573V, K573A, R643W, and R643A were $30 \mu \mathrm{M}, 47$ $\mu \mathrm{M}, 50 \mu \mathrm{M}, 24 \mu \mathrm{M}$, and $60 \mu \mathrm{M}$, respectively. Commercially available c-di-GMP (BioLog) was prepared at a nominal concentration of $500 \mu \mathrm{M}$ in the same solution containing the sample proteins. The interaction between purified samples and c-di-GMP was identified with a Nano ITC (TA Instruments). An aliquot of $2 \mu \mathrm{L}$ of c-di-GMP solution was injected 25 times into the cell filled with the solution containing each 
sample. Stirring velocity was adjusted at $150 \mathrm{rpm}$. Whereas temperature inside the cell was set at $15^{\circ} \mathrm{C}$ in the case of titration of c-di-GMP to all mutants, measurements using wild-type $A x \mathrm{CeSA}-\mathrm{PilZ}$ were performed at $20^{\circ} \mathrm{C}$. All data were fitted to a one-site independent binding curve using NanoAnalyze (TA Instruments). Heat generated from the dilution of c-di-GMP and protein was subtracted prior to data analysis.

\section{Results and discussion}

3.1 Overall structure of $A x \mathrm{CeSA}-\mathrm{PilZ}$

As the Pfam [24] server assigns AxCeSA-PilZ to the domain composed of 99 residues from 572 to 670 in $A x C e S A$ protein (NCBI accession number: BAC82543.1). We over-expressed the domain as a recombinant protein in E.coli and determined the crystal structure of AxCeSA-PilZ. There were two molecules of AxCeSA-PilZ in the asymmetric unit with the orthorhombic space group $P 2{ }_{1} 2_{1} 2_{1}$, having similar conformations with a root mean square deviation (RMSD) of $0.548 \AA$ for $98 \mathrm{C}_{\alpha}$ atoms. The final model of one monomer (molA) in the asymmetric unit comprised 102 residues that are divided into the $A x \mathrm{CeSA}-\mathrm{PilZ}$ region (Gln572-Gly670) and the extra C-terminal 
region (Leu-Glu-His) encoded by the $3^{\prime}$ restriction enzyme site and hexahistidine tag. The first 4 residues of the other monomer (molB) in the asymmetric unit were not modeled because of a lack of significant electron density, and consequently 98 residues (Ser576-Gly670 and the C-terminal Leu-Glu-His) were built. The last three residues of both models became a part of the C-terminal $\alpha$-helix ( $\alpha 1)$ in AxCeSA-PilZ. The overall structure of $A x$ CeSA-PilZ adopts a six-stranded $\beta$-barrel ( $\beta 1-\beta 6)$ flanked by the helix $\alpha 1$ (Fig. 1). The $\beta 2$ and $\beta 5$ strands are curved and participated in two distinct $\beta$-sheets forming a $\beta$-barrel core; i.e., antiparallel $\beta 2-\beta 1-\beta 4-\beta 5$ ( $\beta$-sheet 1 ) and $\beta 2-\beta 3-\beta 6-\beta 5$ ( $\beta$-sheet 2) sheets. A structural similarity search using the DALI [25] server showed that the overall structure of $A x$ CeSA-PilZ resembles those of PilZ domains adopting the six-stranded $\beta$-barrel core, such as PA4608, VCA0042, and PP4397, with Z-scores of 10.5, 10.4, and 10.1, respectively. On the other hand, the structures of type IV fimbriae assembly proteins (PDB: 3CNR, 3DSG) [26,27] the topologies of which are the five-stranded $\beta$-barrel cores were less similar to that of AxCeSA-PilZ with Z-scores of 6.0 and 5.8, respectively.

The surface of $A x$ CeSA-PilZ has a distinctly bipolar nature (Fig. 1). The surface 
formed by $\beta$-sheet 1 is negatively charged, whereas the positive charge on $\mathrm{N}$-terminal loop and $\beta$-sheet 2 including basic residues Lys573, Arg574, His577, Arg578, Lys617, Arg640, Arg643, and Arg648 are widely distributed on the opposite side of the acidic surface. This positively charged surface contributes to electrostatic interaction with c-di-GMP, as the basic region to bind c-di-GMP was found in other PilZ proteins $[14,28]$.

\subsection{Binding properties of c-di-GMP to $A x$ CeSA-PilZ}

Previous studies indicated that not only arginine residues in the Rxxx motif but also the other two residues were involved in binding of c-di-GMP [13,14]. Arg8 and Trp77 in PA4608 and Arg122 and His201 in PP4397 interact directly with guanine bases of c-di-GMPs. Lys573 and Arg643 in AxCeSA-PilZ are regarded as the corresponding residues based on sequence similarity analysis and structure comparison. We designated the positions where Lys573 and Arg643 in AxCeSA-PilZ placed as Position X and Position Y, respectively. To confirm whether Lys573 and Arg643 in AxCeSA-PilZ were involved in recognition of c-di-GMP, the mutants K573V, K573A, R643W, and R643A 
as well as wild-type $A x \mathrm{CeSA}$-PilZ were prepared for the binding analysis using ITC.

The raw ITC data represented that the binding of c-di-GMP to all prepared proteins was exothermic (Fig. 2). The integrated binding isotherm obtained from the experimental data determined the binding stoichiometry of c-di-GMP to each prepared protein and thermodynamic profiles of ligand binding (Table 2). The thermodynamic profiles indicated that the binding of c-di-GMP to all prepared proteins was commonly driven by the favorable enthalpy change with a dissociation constant $K_{\mathrm{d}}$ of submicromolar order. When the structure of $A x C e S A-P i l Z$ was superposed on the known structures of PilZs in complex with c-di-GMP, Lys573 and Arg643 were too far to bind c-di-GMPs (Fig. 3). The enthalpy favorable binding of c-di-GMP observed with ITC supports the conformational rearrangement of $\mathrm{N}$-terminal loop and the reorientation of the side chain of Arg643. Especially, the conformational change in the loop corresponding to the N-terminal loop of AxCeSA-PilZ has been found in other PilZ proteins (VCA0042, PP4397, and PA4608).

These profiles also indicated that the numbers of bound c-di-GMP per molecule of a sample protein were different. The wild-type AxCeSA-PilZ bound two molecules of 
c-di-GMP similar to PP4397 and PA4608. The Position X mutants K573V, which resembles VCA0042, and K573A bound one molecule of c-di-GMP. Moreover, one of the Position Y mutants, R643W, which mimics PA4608, has the capacity to bind two molecules of c-di-GMP. The other mutant R643A, however, bound c-di-GMP with a stoichiometric ratio of 1:1.5 with AxCeSA-PilZ and c-di-GMP.

\subsection{Binding model of c-di-GMP to $A x \mathrm{CeSA}$-PilZ}

Previous reports indicated that the residue at Position $\mathrm{X}$ affects the binding of c-di-GMP $[13,14]$. When arginine occupies Position X, such as Arg8 in PA4608 and Arg122 in PP4397, these proteins are capable of binding dimeric c-di-GMP. In cases in which leucine (Leu135) is present on Position X in VCA0042, the side chain of Leu135 prevented binding to two molecules of c-di-GMP due to steric hindrance between the leucine and guanine base [12]. Furthermore, the structures of PA4608 and PP4397 in complex with c-di-GMP indicated that Trp77 in PA4608 and His201 in PP4397 located at Position $\mathrm{Y}$ made contact with guanine bases. The present study confirmed that AxCeSA-PilZ also captured two molecules of c-di-GMP, where Lys573 and Arg643 
were located at Positions $\mathrm{X}$ and $\mathrm{Y}$, respectively.

Based on the results of binding assays and known structures of PilZs, we propose a manner of interaction between c-di-GMP and PilZ, and a schematic model is shown in Fig. 4. This model includes four residues (two arginine residues in the RxxxR motif and the residues on Positions $\mathrm{X}$ and $\mathrm{Y}$ ) and four guanine bases in two bound c-di-GMPs, arranged in four layers and two columns. G1a-Position X, Arg I-G2b, Arg II-G1b, and G2a-Position Y comprise four layers, whereas G1a-Arg I-Arg II-G2a and Position X-G2b-G1b-Position Y comprise two columns (Fig. 4). Hydrogen bonds between a guanine base and an amino acid in the same layer are formed, whereas stacking or cation- $\pi$ interactions between a guanine base and a residue in the same column are formed. The residues at Position $\mathrm{X}$ and Position $\mathrm{Y}$ as well as the two conserved arginine residues in the RxxxR motif accomplish this symmetrical binding.

The cation- $\pi$ interaction between Lys573 in AxCeSA-PilZ and guanine base G2b may disappear, whereas hydrogen bonding interactions with guanine base G1a may remain, as the corresponding arginine residues of PA4608 and PP4397 were involved in binding of G1a. These observations indicated that hydrogen bonds are critical to bind two 
molecules of c-di-GMP, and cation- $\pi$ interaction assists in the binding of c-di-GMP.

The decreased stoichiometric ratio of 1:1 measured for K573V as well as K573A variants implies that a lack of specific interactions with the residue rather than steric hindrance with the bulky residue at Position X prevents the docking of two mutually intercalated c-di-GMP. The residue at Position Y is also expected to be a key residue for recognizing c-di-GMP. In the case of AxCeSA-PilZ, Arg643 at Position Y may recognize guanine bases G1b and G2a by cation- $\pi$ interaction and by hydrogen bonding interaction, respectively. Although Arg643 in AxCeSA-PilZ was substituted to tryptophan, the mutant R643W could still bind c-di-GMP with invariant stoichiometry. However, the alanine-substituted mutant R643A lacking the ability to interact with both G1b and G2a was probably unable to bind mutually intercalated c-di-GMP dimer, according to the change of stoichiometry from 1:2 to 1:1.5 with AxCeSA-PilZ and c-di-GMP.

\section{Acknowledgment}

We thank the staff of beamline BL-5A at Photon Factory for assistance with data 
collection 


\section{Reference}

[1] J.W. Hickman, C.S. Harwood, Identification of FleQ from Pseudomonas aeruginosa as a c-di-GMP-responsive transcription factor. Mol Microbiol. 69 (2008) 376-389.

[2] F. Tao, Y.W. He, D.H. Wu, S. Swarup, L.H. Zhang, The cyclic nucleotide monophosphate domain of Xanthomonas campestris global regulator Clp defines a new class of cyclic di-GMP effectors. J. Bacteriol. 192 (2010) 1020-1029.

[3] A. Duerig, S. Abel, M. Folcher, M. Nicollier, T. Schweda, N. Amiot, B. Giese, U. Jenal, Second messenger-mediated spatiotemporal control of protein degradation regulates bacterial cell cycle progression. Genes Dev. 23 (2009) 93-104.

[4] C. Matthias, C. Beat, G.A. Martin, F. Marc, J. Paul, G. Stephan, J. Urs, DgrA is a member of a new family of cyclic diguanosine monophosphate receptors and controls flagellar motor function in Caulobacter crescentus. Proc. Natl. Acad. Sci. USA. 104 (2007) 4112-4117.

[5] N. Kulshina, N.J. Baird, A.R. Ferré-D’Amaré, Recognition of the bacterial second messenger cyclic diguanylate by its cognate riboswitch. Nat. Struct. Mol. Biol. 16 (2009) 1212-1217. 
[6] K.D. Smith, S.V. Lipchock, T.D. Ames, J. Wang, R.R. Breaker, S.A. Strobel,

Structural basis of ligand binding by a c-di-GMP riboswitch. Nat. Struct. Mol. Biol. 16

(2009) 1218-1223.

[7] S. Ouyang, X. Song, Y. Wang, H. Ru, N. Shaw, Y. Jiang, F. Niu, Y. Zhu, W. Qiu, K. Parvatiyar, Y. Li, R. Zhang, Z.J. Liu, Structural analysis of the STING adaptor protein reveals a hydrophobic dimer interface and mode of cyclic di-GMP binding. Immunity. 29 (2012) 1073-1086.

[8] D.A. Ryjenkov, M. Tarutina, O.V. Moskvin, M. Gomelsky, Cyclic diguanylate is a ubiquitous signaling molecule in bacteria: insights into biochemistry of the GGDEF protein domain. J. Bacteriol. 187 (2005) 1792-1798.

[9] M. Christen, B. Christen, M. Folcher, A. Schauerte, U. Jenal, Identification and characterization of a cyclic di-GMP-specific phosphodiesterase and its allosteric control by GTP. J. Biol. Chem. 280 (2005) 30829-30837.

[10] R.P. Ryan, Y., Fouhy, J.F. Lucey, L.C. Crossman, S. Spiro, Y.W. He, L.H. Zhang, S. Heeb, M. Cámara, P. Williams, J.M. Dow, Cell-cell signaling in Xanthomonas campestris involves an HD-GYP domain protein that functions in cyclic di-GMP 
turnover. Proc. Natl. Acad. Sci. USA. 25 (2006) 6712-6717.

[11] D. Amikam, M.Y. Galperin, PilZ domain is part of the bacterial c-di-GMP binding protein. Bioinformatics. 22 (2006) 3-6.

[12] J. Benach, S.S. Swaminathan, R. Tamayo, S.K. Handelman, E. Folta-Stogniew, J.E. Ramos, F. Forouchar, H. Neely, J. Seetharaman, A. Camilli, J.F. Hunt, The structural basis of cyclic diguanylate signal transduction by PilZ domains. EMBO J. 26 (2007) $5153-5166$.

[13] J. Ko, K.S. Ryu, H. Kim, J.S. Shin, J.O. Lee, C. Cheong, B.S. Choi, Structure of PP4397 reveals the molecular basis for different c-di-GMP binding modes by Pilz domain proteins. J. Mol. Biol. 23 (2010) 97-110.

[14] J. Habazettl, M.G. Allan, U. Jenal, S. Grzesiek, Solution structure of the PilZ domain protein PA4608 complex with cyclic di-GMP identifies charge clustering as molecular readout. J. Mol. Biol. 22 (2011) 14304-14314.

[15] S. Kawano, K. Tajima, Y. Uemori, H. Yamashita, T. Erata, M. Munekata, M. Takai, Cloning of cellulose synthesis related genes form Acetobacter xylinum ATCC23769 and ATCC53582: comparison of cellulose synthetic ability between strains. DNA. Res. 9 
(2002) 149-156.

[16] S.Q. Hu, Y.G. Gao, K. Tajima, N. Sunagawa, Y. Zhou, S. Kawano, T. Fujiwara, T.

Yoda, D. Shimura, Y. Satoh, M. Munekata, I. Tanaka, M. Yao, Structure of bacterial cellulose synthase subunit D octamer with four inner passageways. Proc. Natl. Acad. Sci. USA. 107 (2010) 17957-17961.

[17] H. Weinhouse, S. Sapir, D. Amikam, Y. Shilo, G. Volman, P. Ohana, M. Benziman, c-di-GMP-binding protein, a new factor regulating cellulose synthesis in Acetobacter xylinum. FEBS Lett. 416 (1997) 207-211.

[18] D. Amikam, M. Benziman, Cyclic diguanylic acid and cellulose synthesis in Agrobacteriumtumefaciens. J. Bacteriol. 171 (1989) 6649-6655.

[19] D.A. Ryjenkov, R. Simm, U. Romling, M. Gomelsky, The PilZ domain is a receptor for the second messenger c-di-GMP: the PilZ domain protein YcgR controls motility in enterobacteria. J. Biol. Chem. 281 (2006) 30310-30314.

[20] W. Kabsch, XDS. Acta. Cryst. D. 66 (2010) 125-132.

[21] P.D. Adams, P.V. Afonine, G. Bunkoczi, V.B. Chen, I.W. Davis, N. Echols, J.J. Headd, L.W. Hung, G.J. Kapral, R.W. Grosse-Kunstleve, A.J. McCoy, N.W. Moriarty, R. 
Oeffner, R.J. Read, D.C. Richardson, J.S. Richardson, T.C. Terwilliger, P.H. Zwart, PHENIX: a comprehensive Python-based system for macromolecular structure solution. Acta Cryst. D. 66 (2010) 213-221.

[22] P. Emsley, K. Cowtan, Coot: model-building tools for molecular graphics. Acta. Cryst. D. 60 (2004) 2126-2132.

[23] V.B. Chen, W.B. Arendall. 3rd, J.J. Headd, D.A. Keedy, R.M. Immormino, G.J. Kapral, L.W. Murray, J.S. Richardson, D.C. Richardson Molprobity: all-atom structure validation for macromolecular crystallography. Acta. Cryst. D. 66 (2010) 12-21.

[24] M. Punta, P.C. Coggill, R.Y. Eberhardt, J. Mistry, J. Tate, C. Boursnell, N. Pang, K. Forslund, G. Ceric, J. Clements, A. Heger, L. Holm, E.L. Sonnhammer, S.R. Eddy, A. Bateman, R.D. Finn, The Pfam protein families database. Nucleic Acids Res. 40 (2012) 290-301.

[25] L. Holm, C. Sacder, DALI: A network tool for protein structure comparison. Trends Biochem Sci. 20 (1995) 478-480.

[26] C.R. Guzzo, R.K. Salinas, M.O. Andrade, C.S. Farah, PILZ Protein Structure and Interactions with PILB and the FIMX EAL Domain: Implications for Control of Type 
IV Pilus Biogenesis. J.Mol.Biol. 393 (2009) 848-866.

[27] T.N. Li, K.H. Chin, J.H. Liu, A.H. Wang, S.H. Chou, XC1028 from Xanthomonas campestris adopts a PilZ domain-like structure without a c-di-GMP switch. Proteins. 75 (2009) 282-288.

[28] K.H. Chin, W.T. Kuo, Y.J. Yu, Y.T. Liao, M.T. Yang, S.H. Chou, Structural polymorphism of c-di-GMP bound to an EAL domain and in complex with a type II PilZ-domain protein. Acta. Cryst. D. 68 (2012) 1380-1392.

\section{Figure legends}

Fig. 1 The overall structure of AxCeSA-PilZ (upper left) and topology of AxCeSA-PilZ (upper right). Acidic (red) and basic (blue) regions were mapped onto the surface of AxCeSA-PilZ. (lower part)

Fig. 2 Binding of c-di-GMP to AxCeSA-PilZ was analyzed by ITC. Upper panels correspond to titration kinetics. Lower panels show the integrated binding isotherms. Molar ratio refers to protein monomer. The binding isotherm of each AxCeSA-PilZ 
titrated with c-di-GMP was corrected with the heat of dilution of the protein solution before analysis.

Fig. 3 Superposed model of AxCeSA-PilZ in complex with c-di-GMP. PilZ domains of VCA0042, PP4397 and PA4608 were superposed on the structure of AxCeSA-PilZ and bound c-di-GMPs are shown in orange and blue. Residues are labeled and represented as yellow (AxCeSA-PilZ), pink (VCA0042), green (PP4397), and light blue (PA4608) sticks.

Fig. 4 Schematic diagram of general interaction manner between dimeric c-di-GMP and PilZs. Two molecules of c-di-GMP are shown in orange and blue. Especially, base parts are depicted by ovals (G1a, G1b, G2a, and G2b). The dotted line, red arrows, and green squares represent hydrogen bonds, cation- $\pi$ interactions, and stacking interactions, respectively. The positions of two arginines conserved in the RxxxR motif and Positions $\mathrm{X}$ and $\mathrm{Y}$ are indicated by circles (magenta). 
Table. 1 Data collection and refinement statistics

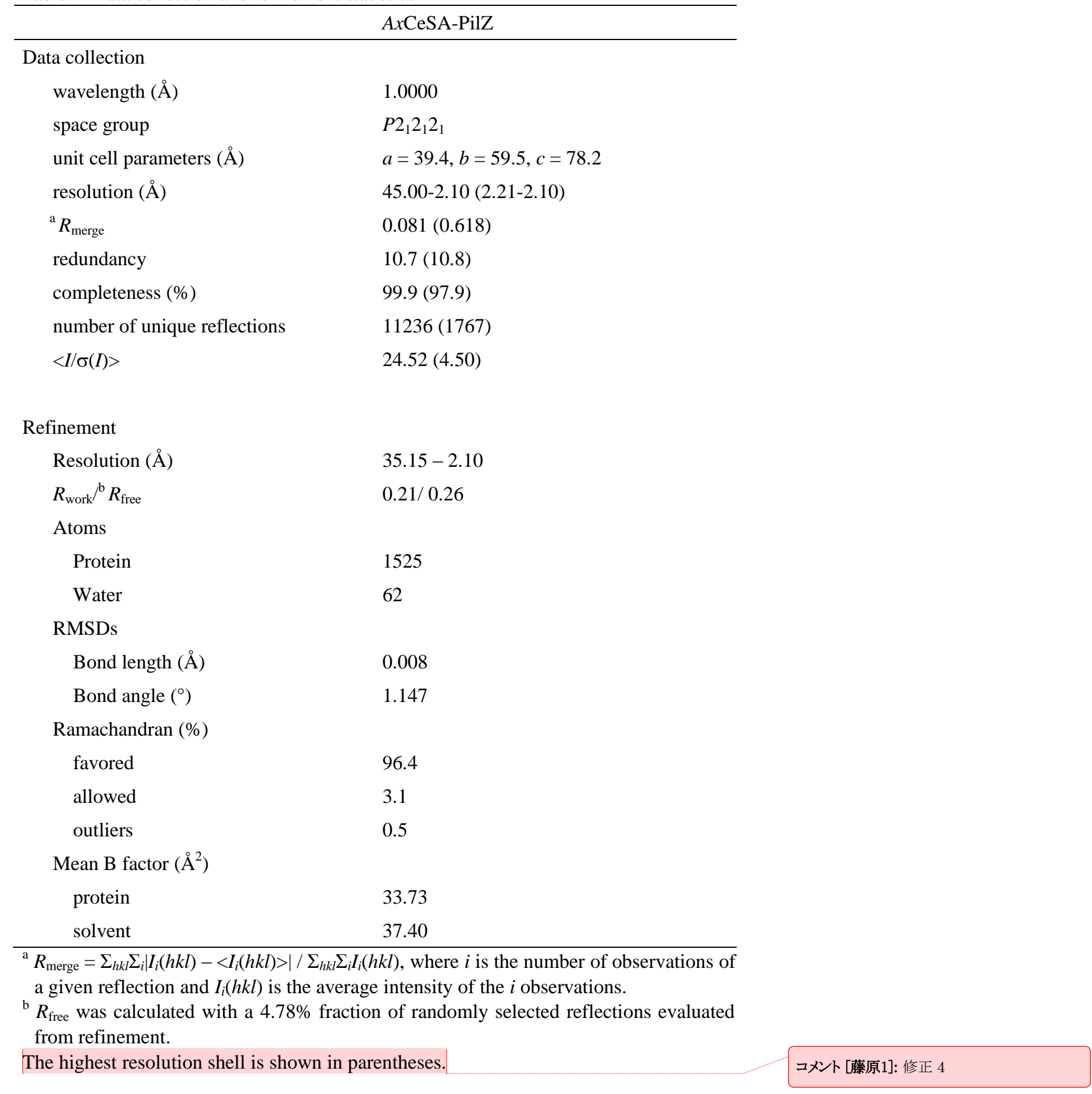


Table 2. Thermodynamic profiles of the binding of c-di-GMP to AxCeSA-PilZ

\begin{tabular}{cccccc}
\hline & w.t. & K573V & K573A & R643W & R643A \\
\hline$N$ & 2.137 & 0.982 & 1.027 & 1.897 & 1.501 \\
$K_{\mathrm{d}}(\mu \mathrm{M})$ & 5.985 & 9.695 & 6.361 & 12.91 & 4.314 \\
$\Delta H\left(\mathrm{~kJ} \mathrm{~mol}^{-1}\right)$ & -36.19 & -53.73 & -39.46 & -40.32 & -32.25 \\
$\Delta S\left(\mathrm{~J} \mathrm{~mol}^{-1} \mathrm{~K}^{-1}\right)$ & -25.66 & -90.59 & -37.47 & -46.42 & -9.208 \\
$\Delta G\left(\mathrm{~kJ} \mathrm{~mol}^{-1}\right)$ & -28.80 & -27.64 & -28.67 & -26.95 & -29.60 \\
\hline
\end{tabular}



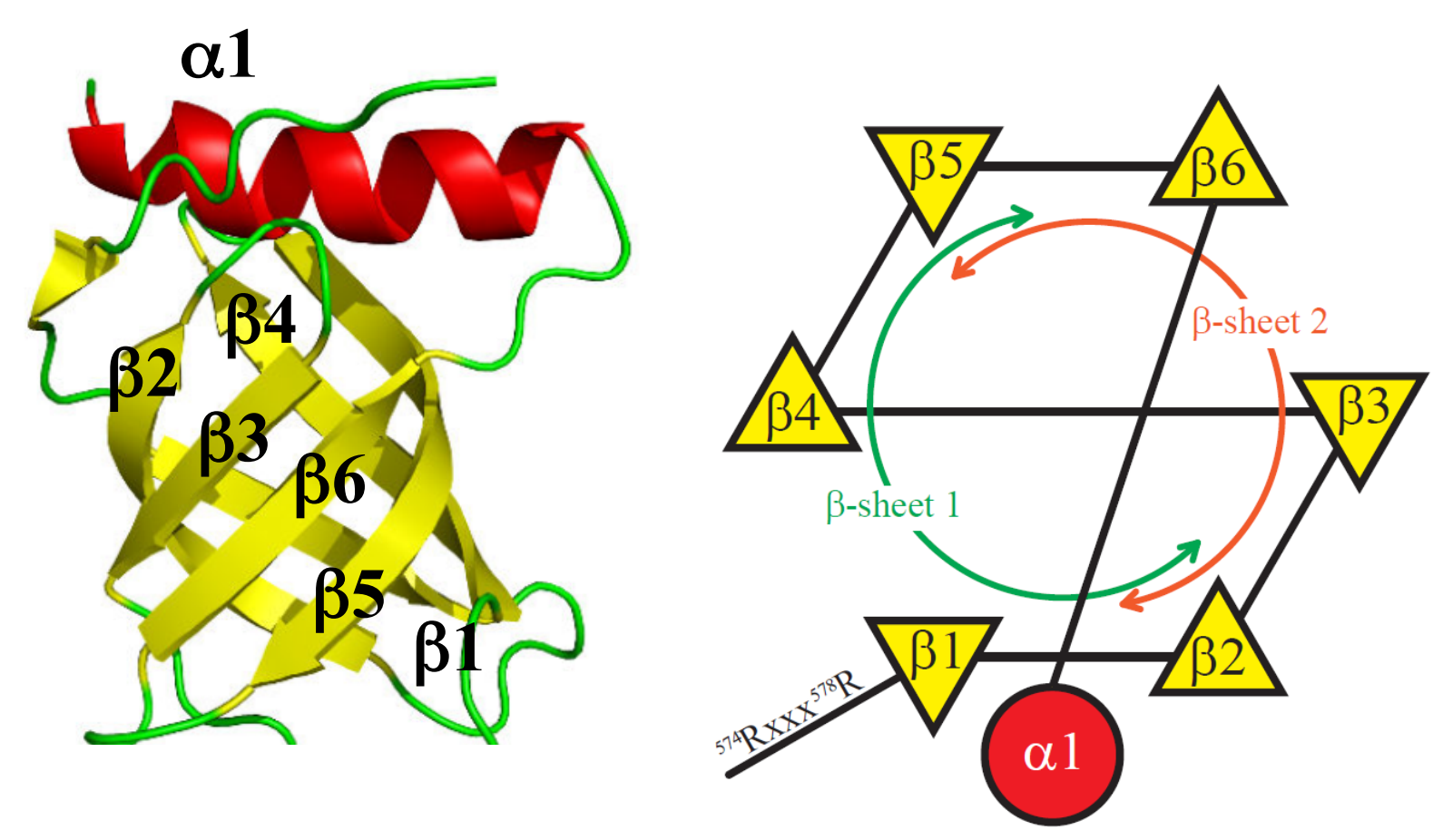

Arg578 Arg574
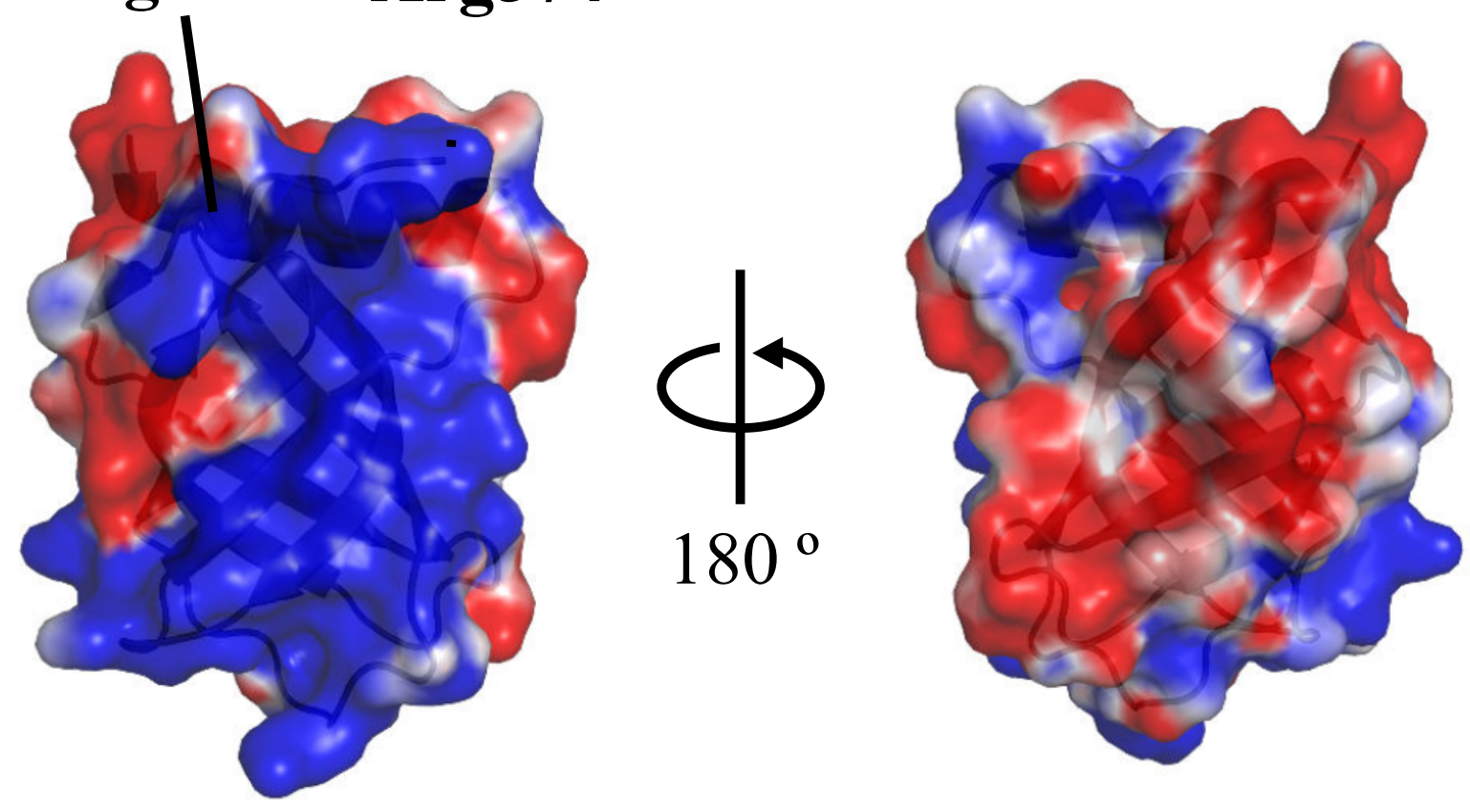


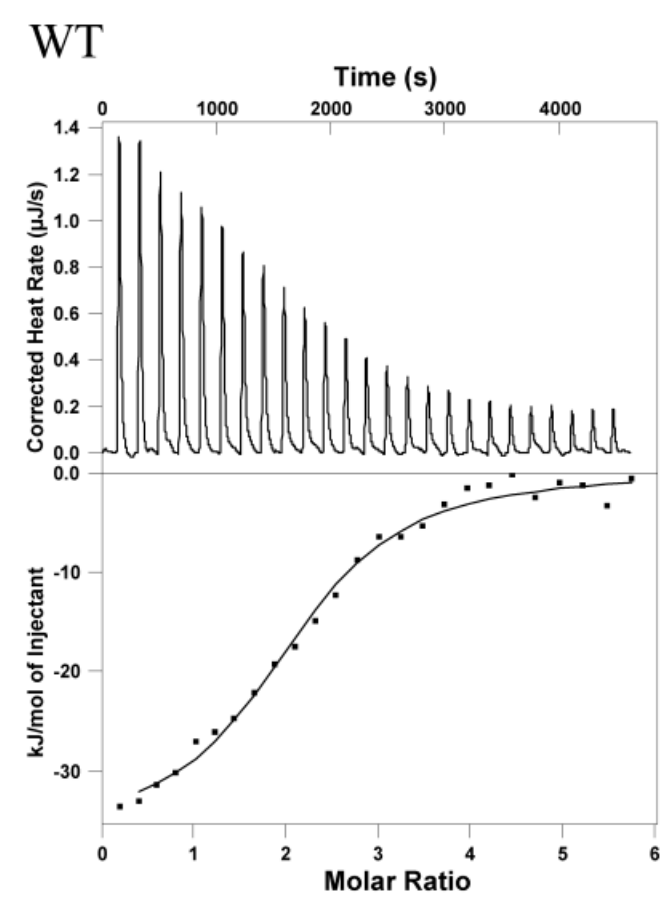

R643W
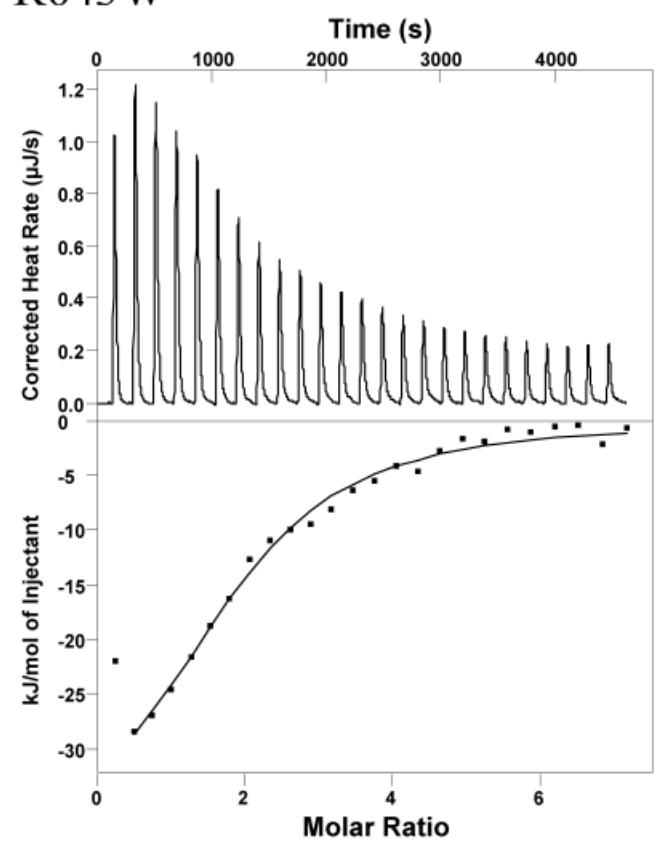

K573V

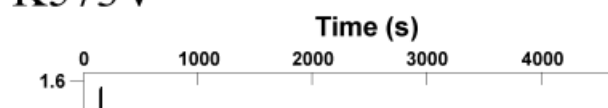
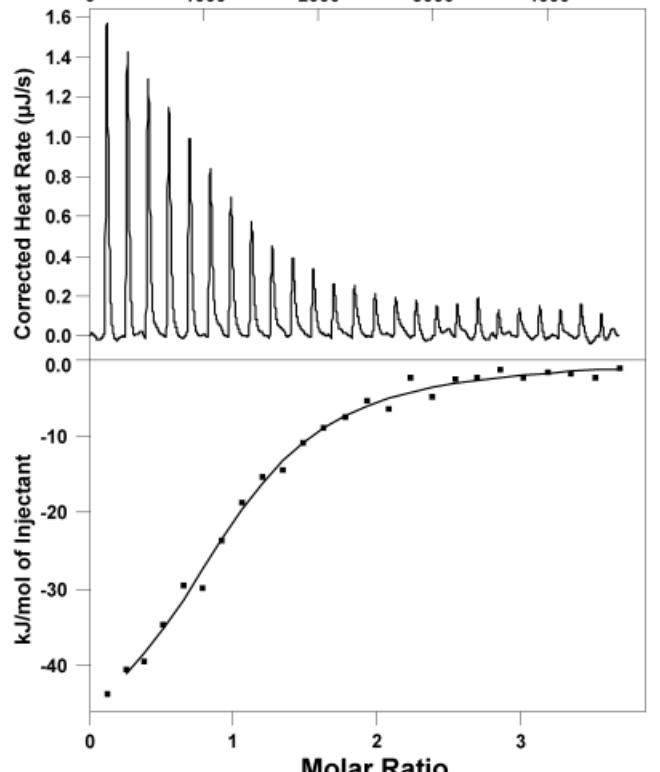

\section{K573A}

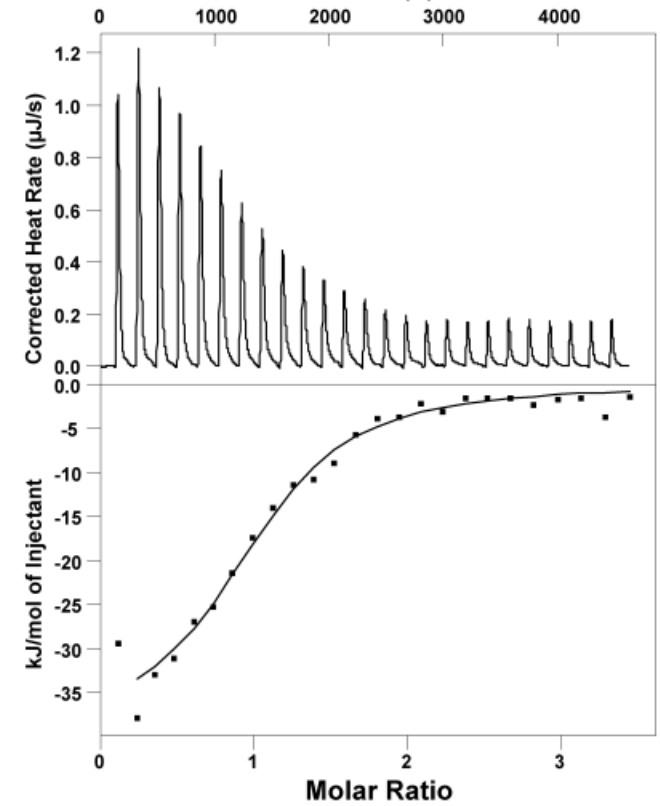

R643A

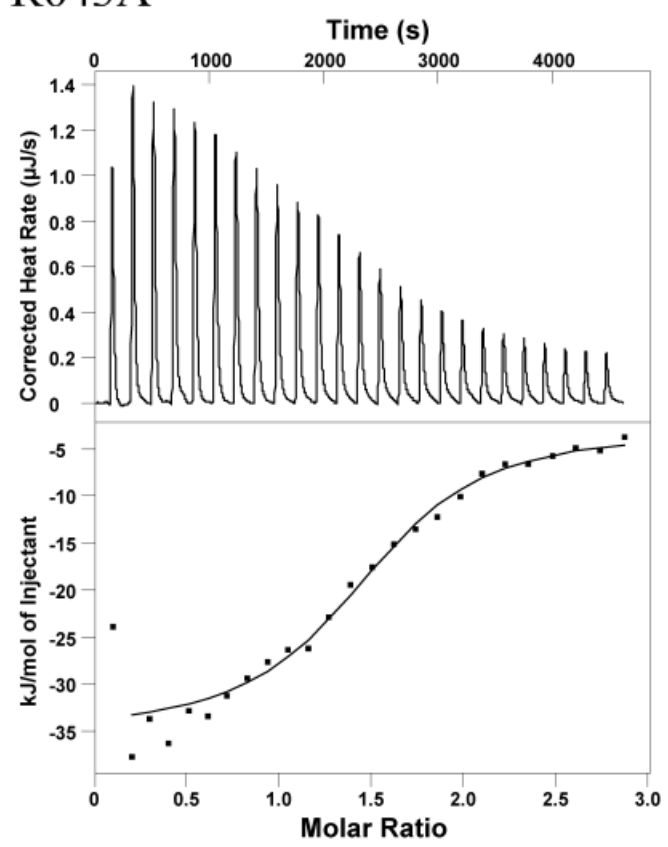




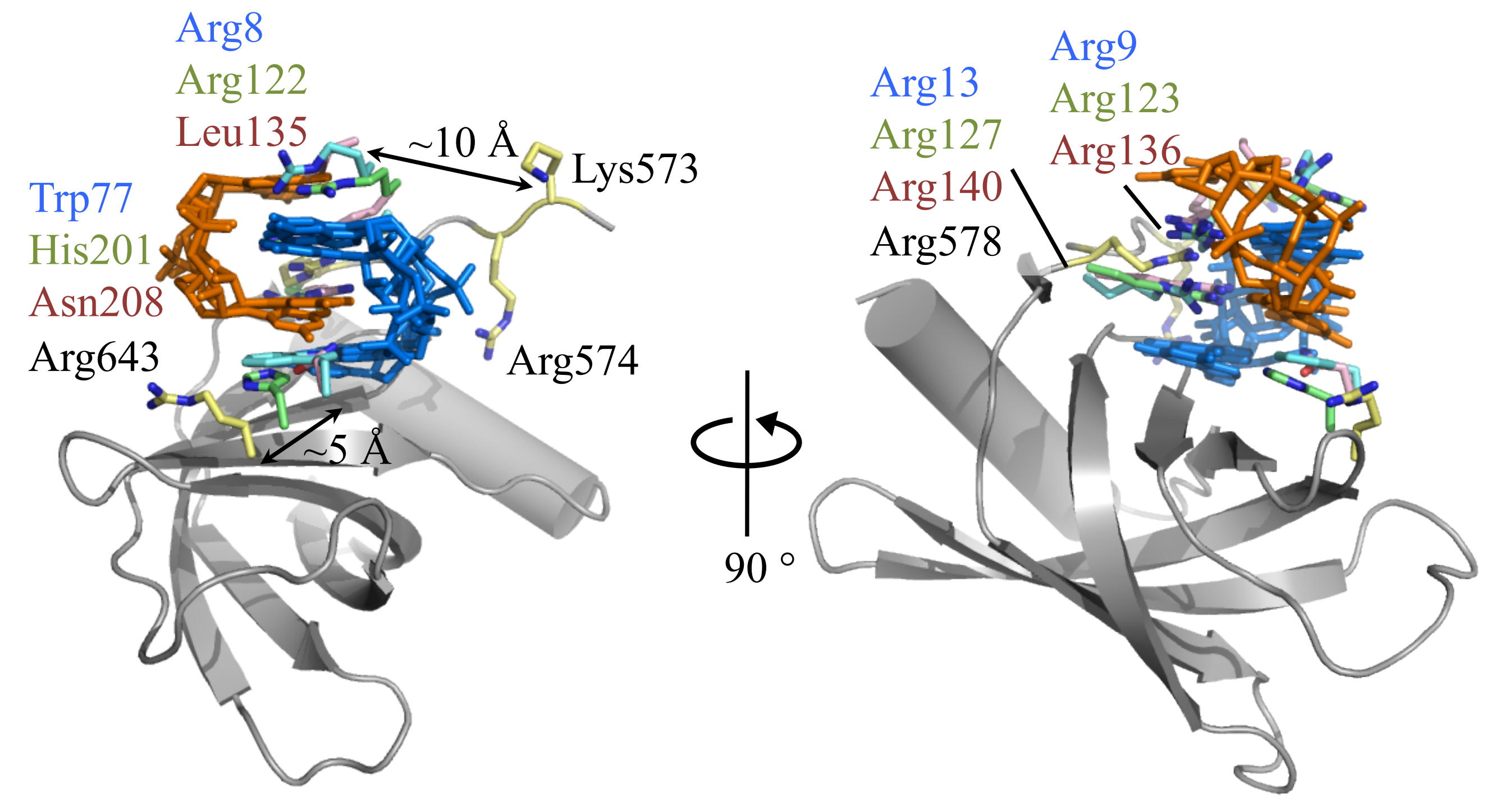




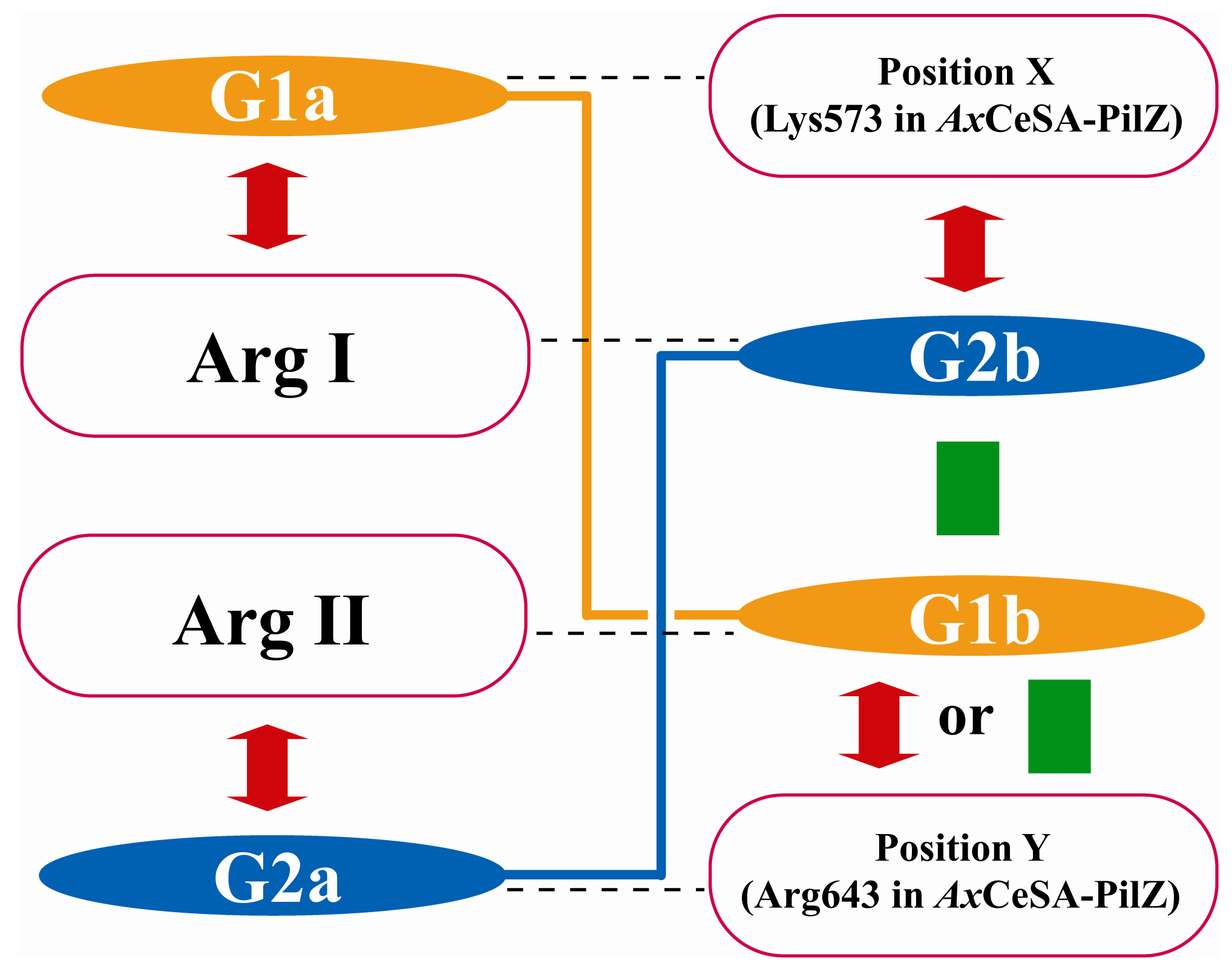

